\title{
Respiratory Care in Neuromuscular Diseases
}

\author{
Ashima S Sahni MD and Lisa Wolfe MD
}

\author{
Introduction \\ Sialorrhea \\ Vocal Cord Spasticity \\ Noninvasive Ventilation \\ Screening for Respiratory Muscle Weakness \\ Initiation of NIV \\ Early vs Late Initiation of NIV \\ Modes of Ventilation \\ Mask Fitting and Desensitization \\ Polysomnogram \\ Monitoring \\ Cough Augmentation \\ End of Life Care \\ Summary
}

\begin{abstract}
Caring for patients with neuromuscular disease (NMD) is challenging. Respiratory care is of the utmost importance because it is a major determinant of quality of life and survival. Noninvasive ventilation (NIV) is one of the few modalities that has shown survival benefit in the NMD patient population. Newer modes with smart technologies are being developed to assist in better ventilation. Some noninvasive methods have shown success in the management of sialorrhea, which is of paramount importance in the initiation of NIV. This review will summarize the management of respiratory symptomatology in patients with NMD with recent advances made in NIV. Key words: ventilation; noninvasive ventilation; neuromuscular weakness; chronic respiratory failure; amyotrophic lateral sclerosis. [Respir Care 2018;63(5):601-608. () 2018 Daedalus Enterprises]
\end{abstract}

\section{Introduction}

Respiratory muscle weakness is common in patients with neuromuscular disease (NMD). ${ }^{1}$ Expiratory, inspiratory,

Dr Sahni is affiliated with the Division of Sleep Medicine, Department of Neurology, Northwestern University Feinberg School of Medicine. Dr Wolfe is affiliated with the Division of Pulmonary and Critical Care Medicine, Department of Medicine, Northwestern University Feinberg School of Medicine.

Dr Wolfe presented a version of this paper at the Year in Review Symposium of the AARC Congress, held October 4-7, 2017, in Indianapolis, Indiana.

The authors have disclosed no conflicts of interest. or upper airway muscles can be compromised, which can lead to chronic respiratory failure. Dyspnea is the most common presentation, although the pathophysiology may vary depending on the location of the lesion (eg, upper vs lower motor neuron lesion). ${ }^{2}$ Noninvasive ventilation (NIV) could prolong survival and improve quality of life. A multidisciplinary approach to symptom management not only

\footnotetext{
Correspondence: Ashima S Sahni MD, Division of Sleep Medicine, Department of Neurology, Feinberg School of Medicine at Northwestern University, 676 N St Clair Street, 7th floor, Chicago, IL 60611. E-mail: 1984ashima@gmail.com.
}

DOI: $10.4187 /$ respcare. 06210 
reduces the hospitalization but also improves the quality of life for these patients.

\section{Sialorrhea}

Sialorrhea refers to excessive salivation caused either due to excessive production of saliva or difficulty in saliva clearance. Patients with NMD develop sialorrhea primarily due to bulbar dysfunction with poor coordination of the tongue and palate. This could result in poor performance with NIV and lead to intolerance of a life-prolonging treatment. Anticholinergics form the bedrock of the management of sialorrhea because they block the cholinergic input to the salivary glands and help in drying up the secretions. The most commonly used medications include topical atropine, oral hyoscyamine sulfate, nebulized or subcutaneous glycopyrrolate, oral amitriptyline, and scopolamine patches. ${ }^{3}$ Atropine and hyoscyamine are efficacious, but they cross the blood-brain barrier and can cause cognitive side effects. Glycopyrrolate is preferred due to a better side effect profile because it doesn't cross the bloodbrain barrier, but it may still cause systemic side effects such as constipation and urinary retention. Amitriptyline has been used orally with much success despite the lack of robust evidence in the literature. Scopolamine patches have shown good response in controlling sialorrhea with efficacy as high as $85 \% .{ }^{4}$ These medications can thicken oral secretions, which can make it difficult to mobilize the secretions. Overall, two thirds of patients respond to these medications, but they may not present a sustainable longterm solution due to unwanted adverse effects.

In patients with refractory sialorrhea, botulinum toxin injection in the salivary gland is viewed as the best alternative. Botulinum toxin blocks the presynaptic release of acetylcholine at the parasympathetic ganglia. Therefore, when injected into the salivary glands, it blocks the production of saliva until the presynaptic terminal regenerates. ${ }^{5}$ Long-term efficacy and safety data with botulinum injection is favorable, with a mean duration of benefit lasting 3.5 months, and after such time the botulinum toxin can be redosed. ${ }^{6}$ The use of ultrasound guidance to assist with localization of the injection may improve the precision of this procedure. ${ }^{7}$

External beam radiation with photon- or electron-based therapy can be used in refractory cases of sialorrhea with lasting benefits. ${ }^{8,9}$ Electron-based therapy is preferred due to its precision in targeting the superficial parotid tissue compared to photon-based therapy..$^{10}$ Radiation is generally preferred over botulinum injection because it doesn't cause a reduction in oropharyngeal function, which is a distinct advantage in patients with bulbar dysfunction. Radiation is not widely accepted due to the perceived side effect of mucositis, although the recent trials do not report it as a potential limitation.

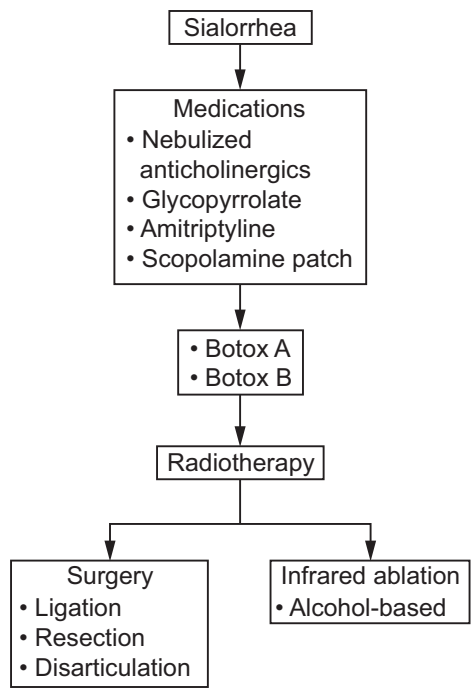

Fig. 1. Management of sialorrhea.

Surgical interventions are considered when conservative approaches have failed. The range of options includes denervation of the salivary glands, excision or ablation of the salivary glands, ligation of the salivary ducts, and relocation of the ducts. The isolated salivary gland ablation, either by excision or ligation, may prove futile due to compensatory hypersecretion from the other salivary glands. ${ }^{11}$ Similarly, the denervation of the salivary glands has proven ineffective due to nerve regeneration. ${ }^{12}$ Recently bilateral submandibulectomy and endoscopic transoral neurectomy of the submandibular gland and sublingual gland have had some success. ${ }^{13}$ Most of these procedures require pulmonary reserve, which is a limitation in adult neuromuscular patients unless they have longstanding use of tracheostomy or mechanical ventilation. Salivary duct ablation could be attempted with alcohol by interventional radiology, although this technique lacks any significant published detail. Figure 1 summarizes the various management options available for these patients.

\section{Vocal Cord Spasticity}

Vocal cord spasticity is seen more commonly in patients with amyotrophic lateral sclerosis (ALS), but it is also seen in patients with spinal muscular atrophy and other neuromuscular conditions. It is frequently reported as a complication in otolaryngology literature, with incidence varying from $4 \%$ to $30 \% .{ }^{14}$ Acute onset of dyspnea or stridor in patients with vocal cord spasticity warrants urgent airway management or tracheotomy. Some patients may report nocturnal stridor as the presenting complaint. This is related to the increase in upper airway resistance leading to increases in negative intra-thoracic pressure during inspiration, which contributes to nocturnal stridor. Non- 
invasive techniques of applying positive pressure with CPAP may be helpful in treating it. ${ }^{15}$ Benzodiazepines, such as diazepam and lorazepam intensol, can be used to relax the spastic oropharyngeal muscles by presynaptic inhibition. This has not been formally studied for vocal cord spasm in NMD. ${ }^{16}$ However, given the well described spasticity associated with the upper motor neuron disease, the use of benzodiazepines is a reasonable first step.

\section{Noninvasive Ventilation}

\section{Screening for Respiratory Muscle Weakness}

The accepted standards for accessing respiratory weakness are transdiaphragmatic pressure and esophageal pressure monitoring. These are invasive and labor-intensive measurements, the repetition of which is impractical in a clinic setting. The surrogate markers for diaphragm weakness include maximum inspiratory pressure $\left(\mathrm{P}_{\text {Imax }}\right)$, supine and upright FVC, overnight oximetry, and $\mathrm{P}_{\mathrm{aCO}}$. Some studies have reported supine FVC as the most highly correlated predictor of transdiaphragmatic pressure $\left(\mathrm{R}^{2}=0.76\right)$, although a combination of supine FVC and $\mathrm{P}_{\text {Imax }}$ was better. ${ }^{17}$ Others have concluded that $\mathrm{P}_{\mathrm{Imax}}$ and nocturnal pulse oximetry were more sensitive in detecting early respiratory weakness compared to FVC. ${ }^{18}$ Therefore, no single maneuver is likely sufficient to define hypoventilation. It is suggested that optimum monitoring of all the parameters at regular intervals is best for early detection of diaphragm weakness.

\section{Initiation of NIV}

NMDs are categorized as restrictive thoracic disorders for Medicare reimbursement for NIV or respiratory assist devices. The minimum requirement for the initial coverage includes symptoms suggestive of hypoventilation (Table 1) and any of the following: awake arterial blood gas $\mathrm{CO}_{2}$ levels $\geq 45 \mathrm{~mm} \mathrm{Hg}$, or oxygen saturation $\left(\mathrm{S}_{\mathrm{pO}_{2}}\right)$ of $\leq 88 \%$ for at least $5 \mathrm{~min}$ of nocturnal recording, or $\mathrm{P}_{\text {Imax }}$ $\geq-60 \mathrm{~cm} \mathrm{H}_{2} \mathrm{O}$ or $\mathrm{FVC}<50 \%$ predicted performed in the upright or supine position. ${ }^{19}$ The European guidelines are more tolerant (Table 2). ${ }^{20}$

ATS guidelines for Duchenne muscular dystrophy (DMD) are more liberal, with the following parameters suggesting the timing of initiation of NIV: signs or symptoms of nocturnal hypoventilation (especially patients with FVC $<30 \%$ predicted/FVC $<1.25 \mathrm{~L}$ ), or apnea hypopnea index of $>10 / \mathrm{h}$ or $\geq 4$ episodes of $\mathrm{S}_{\mathrm{pO}_{2}}<92 \%$, or drops of $\mathrm{S}_{\mathrm{pO}_{2}}$ of at least $4 \%$ per hour of sleep or baseline $\mathrm{S}_{\mathrm{pO}_{2}}$ $<95 \%$, and/or end-tidal $\mathrm{CO}_{2}>45 \mathrm{~mm} \mathrm{Hg}$ while awake. ${ }^{21}$

A recent study showed that the frequencies of the respiratory assessments and initiation of respiratory assist devices in patients with DMD is lower than recommended in the guidelines. Pulmonologists and respiratory thera-
Table 1. Symptoms and Signs of Neuromuscular Disorders

\begin{tabular}{ll}
\hline \multicolumn{1}{c}{ Symptoms } & \multicolumn{1}{c}{ Signs } \\
\hline Dyspnea & Tachypnea \\
Nightmares & Nocturnal hypoxemia \\
Morning headaches & Nocturnal hypoventilation \\
Impaired speech & Weak sniff \\
Hallucinations & Poor cough \\
Recurrent pneumonia & Thoraco-abdominal paradox \\
Early satiation at meals & \\
Daytime sleepiness & \\
Confusion/poor daytime concentration & \\
Disturbed sleep & \\
Fatigue & \\
ADHD and learning difficulties & \\
$\quad$ (in children) & \\
\hline ADHD = attention-deficit hyperactivity disorder & \\
\hline
\end{tabular}

pists should collaborate to encourage health care providers caring for patients with DMD to evaluate these patients in timely manner. ${ }^{22}$

\section{Early Versus Late Initiation of NIV}

Figure 2 elaborates a general approach to the initiation of NIV. Early initiation for of NIV has been associated with prolonged survival. A retrospective study showed that early initiation of NIV prolonged the tracheostomyfree survival (median survival $2.7 \mathrm{y}$ vs $1.8 \mathrm{y}$ ). In this study, the early NIV group also had higher $\mathrm{P}_{\text {Imax }}(-50.4 \pm 4.6 \mathrm{~cm}$ $\mathrm{H}_{2} \mathrm{O}$ vs $\left.-28.2 \pm 2.7 \mathrm{~cm} \mathrm{H}_{2} \mathrm{O}, P<.001\right)$ and lower $\mathrm{P}_{\mathrm{aCO}_{2}}$ $(44.9 \pm 1.7 \mathrm{~mm} \mathrm{Hg}$ and $49.3 \pm 2 \mathrm{~mm} \mathrm{Hg})$, emphasizing that multiple parameters should be assessed while evaluating alveolar hypoventilation..$^{23}$ This could be postulated to improve lung compliance, decrease work of breathing at nighttime, increase rest of the fatigued diaphragm, and reduce the severity of respiratory acidosis-related muscle fatigue. Current U.S. guidelines suggest initiation of NIV at $\mathrm{FVC}<50 \%$, and European guidelines suggest initiation at $\mathrm{FVC}<80 \%$, although we anticipate an imminent change in the U.S. guidelines. ${ }^{24}$

\section{Modes of Ventilation}

In neuromuscular patients, ventilator support can be provided with a home mechanical ventilator. Mechanical ventilators for the home are easier to use and have a back-up battery, but they are often bulky and expensive. NIV devices, commonly referred as respiratory assist devices, are more portable and less expensive alternatives that can provide better quality of life.

If a patient with NMD qualifies, he or she can be prescribed NIV without a CPAP trial or a polysomnogram. In 
Table 2. National Institute for Health and Care Excellence Guidelines

\begin{tabular}{|c|c|}
\hline $\mathrm{FVC} / \mathrm{VC}$ & $\mathrm{P}_{\text {Imax }}$ \\
\hline$<50 \%$ of predicted value & $\geq-40 \mathrm{~cm} \mathrm{H}_{2} \mathrm{O}$ \\
\hline \multirow[t]{2}{*}{$\begin{array}{l}<80 \% \text { of predicted value with any symptoms or } \\
\text { signs of respiratory impairment, particularly orthopnea }\end{array}$} & $\begin{array}{l}\geq-65 \mathrm{~cm} \mathrm{H}_{2} \mathrm{O} \text { for men or } \geq-55 \mathrm{~cm} \mathrm{H}_{2} \mathrm{O} \text { for women, plus signs or symptoms of } \\
\text { respiratory impairment }\end{array}$ \\
\hline & Repeated regular tests show a rate of change of $>10 \mathrm{~cm} \mathrm{H}_{2} \mathrm{O}$ per $3 \mathrm{mo}$ \\
\hline \multicolumn{2}{|l|}{$\begin{array}{l}\text { Data from Reference } 20 \\
\mathrm{VC}=\text { vital capacity }\end{array}$} \\
\hline \multicolumn{2}{|l|}{$\begin{array}{l}\mathrm{VC}=\text { vital capacity } \\
\mathrm{PI}_{\max }=\text { maximum inspiratory pressure }\end{array}$} \\
\hline
\end{tabular}

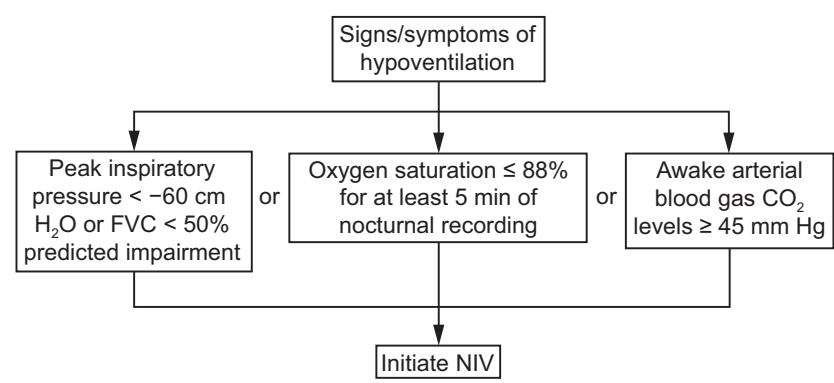

Fig. 2. Initiation of noninvasive ventilation (NIV).

these patients, what may appear to be upper airway resistance on a diagnostic sleep study could be related to diaphragm weakness instead of upper airway narrowing. Bi-level devices can either be used in fixed-pressure or self-adjusting (targeted) pressure modes. Fixed inspiratory positive airway pressure and expiratory positive airway pressure (EPAP) with a back-up rate (NIV in the spontaneous/timed mode) has traditionally been used to care for those with NMD. EPAP is often set as low as possible in neuromuscular patients. The only purpose of EPAP during neuromuscular disease is to flush $\mathrm{CO}_{2}$ from the circuit or to address obstructive sleep apnea in the patient. For concomitant obstructive sleep apnea, EPAP is titrated in the usual manner. Recently, however, newer technology has become available. Patients with progressive disease benefit from variable pressure support as their respiratory efforts may change throughout the day and as the disease advances. Volume-assured pressure support (VAPS) bilevel devices target the alveolar ventilation or exhaled tidal volume and work in the self-adjusting pressure mode, which is an option for these patients. Given that the neuromuscular patient will always require a sufficient minimum level of ventilation, VAPS devices should always be set to assure that even the minimum pressure support level is adequate for the patient's baseline need. The addition of VAPS settings should be thought of as a safety net allowing for higher pressure support when needed. The VAPS device should not be set with a low starting pressure support because the device could undershoot the patient's needs. Once the type of device is chosen, clinicians need to determine how the breath needs to be delivered (timed, spontaneous, or spontaneous/timed). Please refer to Table 3 for further description of NIV modes, and keep in mind these points: add a back-up rate, use appropriate $T_{i}$ min, consider pressure control, and never use adaptive servo ventilation, auto bi-level positive airway pressure, or CPAP. NIV could be used in a spontaneous mode (breaths triggered by the patient only), but this is usually associated with apnea due to respiratory muscle weakness related to failure to trigger. A back-up rate prevents this. A detailed protocol on selecting the appropriate settings with each NIV mode has been discussed in depth elsewhere. ${ }^{25}$

Adaptive sero ventilation is a form of NIV that is used for stabilizing of Cheyne-Stokes respiration. This is not appropriate for patients with NMD.

\section{Mask Fitting and Desensitization}

There are many challenges to fitting a mask in patients with NMD. Neuromuscular weakness can involve the masseter muscle, which can lead to mouth to remain open during sleep. Second, patients who are initiated on NIV at a younger age may have failure of mid-face development, which makes mask fitting even more challenging. ${ }^{26}$ Therefore, finding the perfect mask interface is essential but difficult. Clinicians could try to use pediatric masks with adult patients or use chin straps with nasal pillows. Elevating the tubing to support the weight of the tubing and prevent the tubing from pulling the mask may minimize the leaks from the mask. Please refer to Table 4 for further management options. Caution needs to be used when applying an oronasal mask because this could paradoxically worsen the upper airway resistance. There is evidence of posterior displacement of the tongue causing partial obstruction of the oropharyngeal airway as seen on a nasal endoscopy during a CPAP titration. ${ }^{27}$ Whereas nasal masks are thought to produce a differential pressure gradient between the nasopharynx and oropharynx, thus causing pneumatic splinting, this pushes the soft palate and the tongue anteriorly away from the posterior pharyngeal wall. ${ }^{28}$ Other drawbacks of using an oronasal mask include increased risk of aspiration, increased risk of aerophagia, limited 
Table 3. Modes of Ventilation

\begin{tabular}{ll}
\hline \hline Modes of Ventilation & \multicolumn{1}{c}{ Interpretation } \\
\hline Spontaneous (S) & $\begin{array}{l}\text { 1. Breaths are patient-triggered, pressure-limited, and flow-cycled. } \\
\text { 2. Breaths are triggered by patient effort. Breathing frequency is determined by the patient. The effort needed to start } \\
\text { a breath is either determined by software or set by the clinician. Devices of different manufactures trigger differently. } \\
\text { 3. Breaths are flow cycled from inhalation to exhalation. This can be determined by the software or the clinician. } \\
\text { Devices of different manufactures cycle differently. }\end{array}$ \\
Pressure control (PC) & $\begin{array}{l}\text { 1. Breaths are patient- or time-triggered, pressure-limited, and time-cycled. } \\
\text { 2. Breaths are triggered by the patient or by the ventilator if the patient becomes apneic. }\end{array}$ \\
3. Breaths are cycled by the set inspiratory time. \\
Combination of above \\
1. Breaths are triggered by the ventilator when the patient's breathing rate falls below the back-up rate. \\
2. Breaths are pressure-supported if triggered by the patient or pressure-controlled if triggered by the ventilator.
\end{tabular}

Table 4. Tips to Enhance Adherence With NIV

1. Use pediatric masks in adult patients.

2. Use chin straps with nasal pillows.

3. Elevate the tubing on a head board or hose lift.

4. Add heated humidity and heated wire circuit for airway dryness.

5. Use education and desensitization techniques.

6. Use saline sprays, nasal steroids, or nasal strips for nasal congestion.

7. Use nasal bridge padding, zinc oxide, strap padding, or steroid cream for rash or interface discomfort.

ability for the patient to call care givers for help/assistance, and increased upper-airway obstruction.

Desensitization to masks could be achieved by allowing the patient to play with the mask during the day, and then use the NIV with the mask for few hours in the beginning, then during naps and subsequently nocturnally. This has been elaborated in great detail in a recent review. ${ }^{29}$

\section{Polysomnogram}

Polysomnography is not needed to diagnose alveolar hypoventilation in most adults, although this can be helpful in children. Titration polysomnograghy can be performed as an overnight study. This helps in determining an end-expiratory airway pressure required to overcome any upper airway obstruction, the appropriate pressure support needed to alleviate hypoventilation and ameliorate signs of respiratory distress (ie, tachypnea). More recently a short PAPNAP ambulatory study ${ }^{30}$ has been proposed as an ambulatory 4-h visit instead of an overnight polysomnogram. In this model, the patients were able to go home with the NIV on the same day, which reduced the time between diagnosis and initiation of a life-prolonging treatment. In the near future, with the advent of auto-titration software, titration may not be needed at all in these patients.

\section{Monitoring}

There are no consensus guidelines for NIV regarding an optimal monitoring strategy, compliance goals, or the best follow-up testing. The parameters elaborated in Table 5 could be monitored through tele-monitoring, but this should not replace the in-person interview. Arterial blood gas monitoring or transcutaneous $\mathrm{CO}_{2}$ monitoring have become much more available and in the future may have a more specific role for routine care.

Resupply of the equipment is very important in the longterm success of NIV. Based on Medicare guidelines, Table 6 outlines the frequency of replacement for various respiratory assist devices. ${ }^{36}$

\section{Cough Augmentation}

Patients with NMD often have difficulty clearing secretions due to poor cough. Effective cough can be measured in the patients by assessing cough peak flow, which is the maximum expiratory flow generated by a patient after a forceful cough. A value $<160 \mathrm{~L} / \mathrm{min}$ is considered ineffective for airway clearance. Cough augmentation can assist in increasing the cough peak flow. This is achieved with the help of breath-stacking, mechanical cough augmentation, or manual chest and abdominal compression (ie, manually assisted cough). Sequential breath-stacking (lung volume recruitment) utilizes a hand-held resuscitator with a 1-way valve, which increases lung volume. As a consequence of lung volume recruitment, atelectasis of the bases is prevented and the chest wall remains more flexible due to an increased range of motion. These factors reduce the work of breathing and improve cough strength in patients with NMD. It has been shown to decrease the number of hospitalizations and to improve voice volume and dyspnea. Manually assisted cough can be administered while a patient is either seated or recumbent at $30^{\circ}$. The patient is asked to take a deep inspiration, and then the provider gives a rapid abdominal thrust below the xyphi- 
Table 5. Monitoring Parameters

\begin{tabular}{|c|c|c|}
\hline Parameters & Value & Possible Solutions \\
\hline $\begin{array}{l}\text { Rapid shallow breathing } \\
\text { index }\left(\mathrm{f} / \mathrm{V}_{\mathrm{T}}\right)\end{array}$ & $<40 \mathrm{~L}^{31}$ & $\begin{array}{l}\text { Check for leaks. } \\
\text { Increase the pressure support. }\end{array}$ \\
\hline Leaks & $\begin{array}{l}\text { Minimum leak, defined as a small leak that includes any } \\
\text { of the following: }{ }^{32} \\
\text { 1. }<1 \mathrm{~h} \text { of large leak (Philips Respironics) } \\
\text { 2. }<24 \mathrm{~L} / \mathrm{min} \text { at } 95^{\text {th }} \text { percentile (ResMed nasal mask) } \\
\text { 3. }<36 \mathrm{~L} / \mathrm{min} \text { at } 95^{\text {th }} \text { percentile (ResMed oronasal mask) } \\
\text { 4. }<60 \mathrm{~L} / \mathrm{min} \text { (Fisher \& Paykel) }\end{array}$ & $\begin{array}{l}\text { Consider using the pressure control mode. } \\
\text { Consider change of mask. }\end{array}$ \\
\hline Exhaled tidal volume & $\begin{array}{l}6-8 \mathrm{~mL} / \mathrm{kg}^{33} \\
\text { Lower values for children or for those with scoliosis or } \\
\text { bulbar dysfunction. } \\
\text { Higher values for those with spinal cord injury or obesity. }\end{array}$ & Increase the pressure support. \\
\hline $\begin{array}{l}\text { Residual apnea hypopnea } \\
\text { index }\end{array}$ & $\begin{array}{l}\text { Not used in neuromuscular patients, only in patients with } \\
\text { concomitant sleep apnea. }\end{array}$ & \\
\hline Duration of usage & $>4 \mathrm{~h}^{34}$ & $\begin{array}{l}\text { If usage is }>12 \mathrm{~h} \text {, consider initiation of mouthpiece } \\
\text { ventilation and/or enrollment in a hospice program. }\end{array}$ \\
\hline Overnight pulse oximetry & $\begin{array}{l}\text { S }>90 \% \text { for } 95 \% \text { of an overnight recoding } 35 \\
\text { Has mortality benefit. }\end{array}$ & \\
\hline
\end{tabular}

Table 6. Resupply Schedule per Medicare Guidelines

\begin{tabular}{ll}
\hline \hline \multicolumn{1}{c}{ Equipment } & \multicolumn{1}{c}{ Schedule } \\
\hline Oral, nasal, nasal pillows, oronasal masks & Once every 3 mo \\
Full face cushion & Once every mo \\
Nasal cushion and pillows & Twice every mo \\
Disposable filters & Twice every mo \\
Headgear and chinstraps & Once every 6 mo \\
Humidifier chamber & Once every 6 mo \\
CPAP tubing & Once every 3 mo \\
Disposable filters & Twice every mo \\
Non disposable filters & Once every 6 mo \\
Oral interface & $90 \mathrm{~d}$ \\
\hline
\end{tabular}

sterum. This increases alveolar and pleural pressures, improving cough peak flow and thus clearance of secretions. ${ }^{37}$ Mechanical in-exsufflation therapy generates a pressurelimited insufflation in the lung followed by a quick reversal of the negative pressure, leading to the generation of expiratory flow sufficient to clear secretions. The pressure setting recommended for mechanical in-exsufflation in NMD patients is $+40 /-40 \mathrm{~cm} \mathrm{H}_{2} \mathrm{O}$ for effective cough. ${ }^{88,39}$ A respiratory therapist at the bedside should also consider the effectiveness of any setting with a mindful eye to the exhaled cough volume and peak flow. Mechanical in-exsufflation has been shown to prevent intubation, tracheostomy, and hospitalizations. Recent literature suggests that the application of higher insufflation pressures may be counterproductive in patients with progression of ALS, especially with the onset of the bulbar symptoms. ${ }^{40}$ The combination of the cough augmentation techniques is recommended because they are able to enhance cough peak flow even further. A clinical approach to cough augmen-

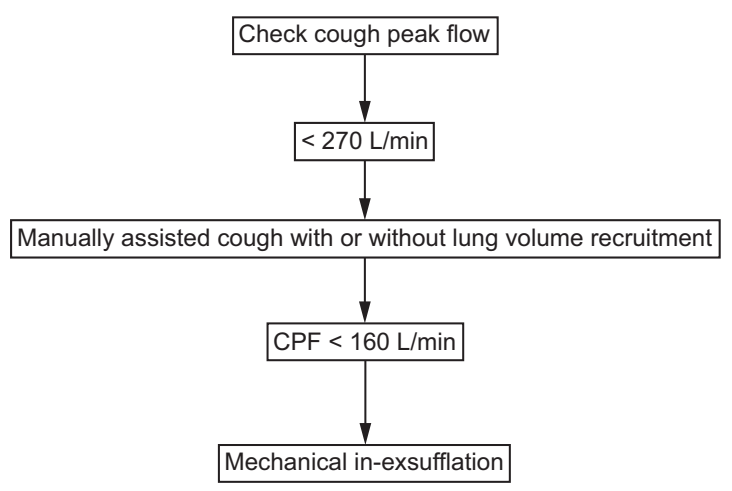

Fig. 3. Approach to cough augmentation.

tation is outlined in Figure 3. A recent systematic review showed weak evidence in support of the continued use of mechanical in-exsufflation in NMD patients; the absence of alternatives makes this a difficult treatment decision. ${ }^{41}$ A randomized, controlled, crossover, single-center trial with 40 subjects with NMD showed that a combination of mechanical in-exsufflation in conjunction with manual thrust improved cough peak flow to a mean of $202.4 \mathrm{~L} / \mathrm{min}$ versus mechanical in-exsufflation alone $(177.2 \mathrm{~L} / \mathrm{min}){ }^{42}$ A randomized, controlled trial showed improved survival at 12 months in subjects enrolled in a breath-stacking group when compared to mechanical in-exsufflation, although the difference was not statistically significant. In a subgroup analysis, improved survival was even seen in patients with severe bulbar dysfunction in the breath-stacking arm. ${ }^{43} \mathrm{~A}$ randomized, controlled trial done in quadriplegic spastic cerebral palsy children showed that mechanical in-exsufflation shortened the duration of therapy for airway clearance when compared to chest physio- 
therapy and was a safe and efficient alternative for airway clearance. ${ }^{44}$ While these techniques are helpful, they have limitations in patients who have severe bulbar paralysis, COPD, or severe chest wall restriction.

High-frequency oscillatory ventilation could also be used to reduce pneumonia and hospitalization. The use of highfrequency oscillatory ventilation in ALS patients has been shown to reduce breathlessness with stabilization of FVC in patients who had FVC of $40-70 \%$ of predicted. ${ }^{45} \mathrm{~A}$ recent cohort study of patients with NMD showed that the total medical costs, hospitalization, and pneumonia after high-frequency oscillatory ventilation were reduced. ${ }^{46}$

\section{End of Life Care}

As an NMD progresses, patients require ventilator support $24 \mathrm{~h} / \mathrm{d}$. It is a common practice to recommend invasive ventilation at this stage, although this increases the frequency of institutional care. Continuous NIV with mouthpiece ventilation during the day and NIV during the night has increased in popularity as a safe alternative in patients with NMD. ${ }^{47}$ This is not a new approach, dating back to post polio patients in 1960s. In a prospective trial done on DMD subjects, 24-h NIV was shown to be a safe alternative in prolonging survival with stabilization of vital capacity. ${ }^{48}$ Mouthpiece ventilation allows patients to be interface free during the day, resulting in better quality of life. Mouthpiece ventilation is best delivered in the volume-cycle mode. Effective airway clearance and the availability of a home caretaker is of great importance in the success of 24-h NIV.

Dyspnea is the most common complaint of ALS patients in hospice. NIV has been shown to alleviate the dyspnea in these patients but can be challenging in patients with severe bulbar dysfunction. Narcotics remain the cornerstone in management. A prospective non-randomized trial involving patients with ALS showed that extendedrelease morphine relieved dyspnea without a significant rise in transcutaneous $\mathrm{CO}_{2}$ levels. ${ }^{49}$ On the other hand supplemental oxygen was not any better than room air in relieving dyspnea in subjects with life-limiting illnesses. ${ }^{50}$ Therefore, given the high risk of $\mathrm{CO}_{2}$ retention as well as the inability to palliate symptoms of dyspnea, supplemental oxygen should not be used in patients with NMD.

\section{Summary}

Supportive care in patients with NMD has demonstrated improved outcomes, and the management of respiratory symptoms is a crucial component that affects quality of life. NIV has shown the largest impact on survival and quality of life in patients with ALS. The advent of a VAPS algorithm as an option for an auto-titration algorithm for NIV will reduce the time between diagnosis and initiation of life-prolonging treatment. The increased popularity of 24-h NIV is likely to improve patient quality of life.

\section{REFERENCES}

1. Perrin C, Unterborn JN, Ambrosio CD, Hill NS. Pulmonary complications of chronic neuromuscular diseases and their management. Muscle Nerve 2004;29(1):5-27.

2. Garg N, Park SB, Vucic S, Yiannikas C, Spies J, Howells J, et al. Differentiating lower motor neuron syndromes. J Neurol Neurosurg Psychiatry 2017;88(6):474-483.

3. Banfi P, Ticozzi N, Lax A, Guidugli GA, Nicolini A, Silani V. A review of options for treating sialorrhea in amyotrophic lateral sclerosis. Respir Care 2015;60(3):446-454.

4. Odachi K, Narita Y, Machino Y, Yamada T, Nishimura Y, Ota Y, et al. Efficacy of transdermal scopolamine for sialorrhea in patients with amyotrophic lateral sclerosis. Cogent Med 2017;4:1365401.

5. Squires N, Humberstone M, Wills A, Arthur A. The use of botulinum toxin injections to manage drooling in amyotrophic lateral sclerosis/motor neurone disease: A systematic review. Dysphagia 2014; 29:500-508.

6. Contarino MF, Pompili M, Tittoto P, Vanacore N, Sabatelli M, Cedrone $\mathrm{A}$, et al. Botulinum toxin $\mathrm{B}$ ultrasound-guided injections for sialorrhea in amyotrophic lateral sclerosis and Parkinson's disease. Parkinsonism Relat Disord 2007;13(5):299-303.

7. Shariat-Madar B, Chun RH, Sulman CG, Conley SF. Safety of ultrasound-guided botulinum toxin injections for sialorrhea as performed by pediatric otolaryngologists. Otolaryngol Head Neck Surg 2016;154(5):924-927.

8. Hawkey NM, Zaorsky NG, Galloway TJ. The role of radiation therapy in the management of sialorrhea: a systematic review. Laryngoscope 2016;126(1):80-85.

9. Assouline A, Levy A, Abdelnour-Mallet M, Gonzalez- Bermejo J, Lenglet T, Forestier NL, et al. Therapy for hypersalivation: A prospective study in 50 amyotrophic lateral sclerosis patients. Int J Radiat Oncol Biol Phys 2014;88(3):589-595.

10. Neppelberg E, Haugen DF, Thorsen L, Tysnes OB. Radiotherapy reduces sialorrhea in amyotrophic lateral sclerosis. Eur J Neurol 2007;14(12):1373-1377.

11. Chaushu G, Dori S, Sela BA, Taicher S, Kronenberg J, Talmi YP. Salivary flow dynamics after parotid surgery: A preliminary report. Otolaryngol Head Neck Surg 2001;124(3):270-273.

12. Greensmith AL, Johnstone BR, Reid SM, Hazard CJ, Johnson HM, Reddihough DS. Prospective analysis of the outcome of surgical management of drooling in the pediatric population: A 10-year experience. Plast Reconstr Surg 2005;116(5):1233-1242.

13. Ozturk K, Erdur O, Gul O, Olmez A. Feasibility of endoscopic submandibular ganglion neurectomy for drooling. Laryngoscope 2017;127(7):1604-1607.

14. Hillel A, Miller R. Bulbar amyotrophic lateral sclerosis: Patterns of progression and clinical management. Head Neck 1989;11(1):51-59.

15. Nonaka M, Imai T, Shintani T, Kawamata M, Chiba S, Matsumoto $H$. Non-invasive positive pressure ventilation for laryngeal contraction disorder during sleep in multiple system atrophy. J Neurol Sci 2006;247(1):53-58.

16. Mathew A, Mathew MC, Thomas M, Antonisamy B. The efficacy of diazepam in enhancing motor function in children with spastic cerebral palsy. J Trop Pediatr 2005;51(2):109-113.

17. Lechtzin N, Wiener CM, Shade DM, Clawson L, Diette GB. Spirometry in the supine position improves the detection of diaphragmatic weakness in patients with amyotrophic lateral sclerosis. Chest 2002;121(2):436-442.

18. Jackson CE, Rosenfeld J, Moore DH, Bryan WW, Barohn RJ, Wrench $\mathrm{M}$, et al. A preliminary evaluation of a prospective study of pulmo- 


\section{Respiratory Care in Neuromuscular Diseases}

nary function studies and symptoms of hypoventilation in ALS/MND patients. J Neurol Sci 2001;191(1-2):75-78.

19. Medicare C. Respiratory assist device: Medical review documentation checklist. https://www.cgsmedicare.com $/ \mathrm{jc} / \mathrm{mr} / \mathrm{pdf} / \mathrm{mr}$ _checklist_rad_ e0471.pdf. Accessed August 3, 2017.

20. National Institute for Health and Care Excellence. Motor neurone disease: Assessment and management (NICE guideline NG42). http:// nice.org.uk/guidance/ng42. Accessed April 4, 2018.

21. Finder JD, Birnkrant D, Carl J, Farber H, Gozal D, Iannaccone ST, et al. Respiratory care of the patient with Duchenne muscular dystrophy: An official ATS consensus statement. Am J Respir Crit Care Med 2004;170:456-465.

22. Andrews J, Soim A, Pandya S, Westfield C, Ciafaloni E, Fox D, et al. Respiratory care received by individuals with Duchenne muscular dystrophy from 2000 to 2011. Respir Care 2016;61(10):13491359.

23. Lechtzin N, Scott Y, Busse A, Clawson L, Kimball R, Wiener C. Early use of non-invasive ventilation prolongs survival in subjects with ALS. Amyotroph Lateral Scler 2007;8:185-188.

24. Andersen PM, Abrahams S, Borasio GD, de Carvalho M, et al. EFNS guidelines on the clinical management of amyotrophic lateral sclerosis (MALS)-revised report of an EFNS task force. Eur J Neurol 2012;19(3):360-375.

25. Selim BJ, Wolfe L, Coleman JM 3rd, Dewan NA. Initiation of noninvasive ventilation for sleep related hypoventilation disorders: advanced modes and devices. Chest 2018;153(1):251-265.

26. Schroth MK. Special considerations in the respiratory management of spinal muscular atrophy. Pediatrics 2009;123(Suppl 4):S245-S249.

27. Schorr F, Genta PR, Gregorio MG, Danzi-Soares NJ, Lorenzi-Filho G. Continuous positive airway pressure delivered by oronasal mask may not be effective for obstructive sleep apnoea. Eur Respir J 2012; 40:503-505.

28. Ng JR, Aiyappan V, Mercer J, Catcheside PG, Chai-Coetzer CL, McEvoy RD, et al. Choosing an oronasal mask to deliver continuous positive airway pressure may cause more upper airway obstruction or lead to higher continuous positive airway pressure requirements than a nasal mask in some patients: A case series. Clin Sleep Med 2016;12(9):1227-1232.

29. Sharma R, Wolfe L. Use of non-invasive ventilation in neuromuscular disease. Curr Sleep Med Rep 2017;3:290.

30. Sheers N, Berlowitz D, Rautela L, Batchelder I, Hopkinson K, Howard M. Improved survival with an ambulatory model of non-invasive ventilation implementation in motor neuron disease. Amyotrophic Lateral Scler Frontotemporal Degener 2014;5(3-4):180-184.

31. Nicholson TT, Smith SB, Siddique T, Sufit R, Ajroud-Driss S, Coleman JM 3rd, et al. Respiratory pattern and tidal volumes differ for pressure support and volume-assured pressure support in amyotrophic lateral sclerosis. Ann Am Thorac Soc 2017;14(7):1139-1146.

32. Schwab R, Badr S, Epstein L, Gay P, Gozal D, Malcolm K, et al. An official American Thoracic Society Statement: Continuous positive airway pressure adherence tracking systems. 2013;188(5):613-620.

33. Berry RB, Chediak A, Brown LK, Finder J, Gozal D, Iber C, et al. Best clinical practices for the sleep center adjustment of noninvasive positive pressure ventilation (NPPV) in stable chronic alveolar hypoventilation syndromes. J Clin Sleep Med 2010;6(5):491-509.

34. Lo Coco D, Marchese S, Pesco MC, La Bella V, Piccoli F, Lo Coco A. Noninvasive positive-pressure ventilation in ALS: Predictors of tolerance and survival. Neurology 2006;67(5):761-765.
35. Bermejo JG, Panzini CM, Arnol N, Meininger V, Kraoua S, Salachas F et al. Prognostic value of efficiently correcting nocturnal desaturations after one month of non-invasive ventilation in amyotrophic lateral sclerosis: A retrospective monocentre observational cohort study. Amyotroph Lateral Scler Frontotemporal Degener, 2013;14: 373-379.

36. DME MAC LCDs: CIGNA Government Services (L11518) dated Feb 4, 2011; National Government Services (L27230), dated Oct 1, 2011; National Heritage Insurance Company (L11504), dated Feb 4, 2011; and Noridian Administrative Services (L171), dated Oct 1, 2011.

37. Rokadia HK, Adams JR, McCarthy K, Aboussouan LS, MirelesCabodevila E. Cough augmentation in a patient with neuromuscular disease. Annals Am Thorac Soc 2015;12(12):1888-1891

38. Schmitt JK, Stiens S, Trincher R, Lam M, Sarkarati M, Linder S. Survey of use of the insufflator-exsufflator in patients with spinal cord injury. J Spinal Cord Med 2007;30(2):127-130.

39. Vianello A, Corrado A, Arcaro G, Gallan F, Ori C, Minuzzo M, et al. Mechanical insufflation-exsufflation improves outcomes for neuromuscular disease patients with respiratory tract infections. Am J Phys Med Rehabil. 2005;84(2):83-88.

40. Andersen T, Sandnes A, Fondenes O, Nilsen R, Tysnes OB, Heimdal $\mathrm{JH}$, et al. Laryngeal responses to mechanically assisted cough in deteriorating amyotrophic lateral sclerosis. Respir Care 2018;63(5): 538-549.

41. Auger C, Hernando V, Galmiche H. Use of mechanical insufflationsexsufflation devices for airway clearance in subjects with neuromuscular disease. Respir Care 2017;62(2):236-245.

42. Kim SM, Choi WA, Won YH, Kang SW. A comparison of cough assistance techniques in patients with respiratory muscle weakness. Yonsei Med J 2016;57(6):1488-1493.

43. Rafiq M, Bradburn M, Proctor A, Billings C, Bianchi S, Mcdermott $\mathrm{C}$, et al. A preliminary randomized trial of the mechanical insufflator-exsufflator versus breath-stacking technique in patients with amyotrophic lateral sclerosis. Amyotroph Lateral Scler Frontotemporal Degener 2015;16:448-455.

44. Siriwat R, Deerojanawong J, Sritippayawan S, Hantragool S, Cheanprapai $\mathrm{P}$. Mechanical insufflation-exsufflation versus conventional chest physiotherapy in children with cerebral palsy. Respir Care 2018;63(2):187-193

45. Lange DJ, Lechtzin N, Davey C, David W, Heiman-Patterson T, Gelinas D, et al. High-frequency chest wall oscillation in ALS: An exploratory randomized, controlled trial. Neurology 2006;67(6):991997.

46. Lechtzin N, Wolfe LF, Frick KD. The impact of high-frequency chest wall oscillation on healthcare use in patients with neuromuscular diseases. Ann Am Thorac Soc 2016;13(6):904-909.

47. McKim D, Griller N, LeBlanc C, Woolnough A, King J. Twentyfour-hour noninvasive ventilation in Duchenne muscular dystrophy: A safe alternative to tracheostomy. Can Respir J 2013;20(1):e5-e9.

48. Toussaint M, Steens M, Wasteels G, Soudon P. Diurnal ventilation via mouthpiece: Survival in end-stage Duchenne patients. Eur Respir J 2006;28:549-555.

49. Clemens KE, Klaschik E. Morphine in the management of dyspnoea in ALS. A pilot study. Eur J Neurol 2008;15:445-450.

50. Abernethy AP, McDonald CF, Frith PA, Clark K, Herndon JE 2nd, Marcello J, et al. Effect of palliative oxygen versus room air in relief of breathlessness in patients with refractory dyspnoea: A doubleblind, randomised controlled trial. Lancet 2010;376:784-793. 